\title{
Philosophiques
}

\section{Vérité. Réalité. Universalité.}

\section{Isabelle Thomas-Fogiel}

Volume 47, numéro 2, automne 2020

URI : https://id.erudit.org/iderudit/1075135ar

DOI : https://doi.org/10.7202/1075135ar

Aller au sommaire du numéro

Éditeur(s)

Société de philosophie du Québec

ISSN

0316-2923 (imprimé)

1492-1391 (numérique)

Découvrir la revue

Citer ce document

Thomas-Fogiel, I. (2020). Vérité. Réalité. Universalité. Philosophiques, 47(2),

457-469. https://doi.org/10.7202/1075135ar d'utilisation que vous pouvez consulter en ligne.

https://apropos.erudit.org/fr/usagers/politique-dutilisation/ 


\title{
Vérité. Réalité. Universalité.
}

\author{
ISABELLE THOMAS-FOGIEL
}

Département de philosophie, Université d'Ottawa

Toute disputatio suppose un opponens qui formule des objections. La tâche est ardue et hardie s'agissant de Bitbol. J'ai déjà indiqué dans une recension (lectio) combien son texte contre le réalisme spéculatif était fondamental; j'ai analysé les arguments des deux partis en légitimant (en dépit de quelques perplexités que je ne souhaite pas répéter ici) la démarche de Bitbol, assumant ainsi le rôle du respondens. Mais Disputatio oblige, il faut maintenant objecter. Pour ce faire, il serait commode d'extraire de ce texte quelques thèmes particuliers qui, indépendamment de l'opposition à Meillassoux, expriment le contenu positif de la philosophie de Bitbol. Mais lesquels choisir, autres que ceux déjà évoqués dans ma recension (l'usage de la contradiction performative, le «je» existentiel, la référence au mystique?). Les analyses abondantes, limpides et passionnantes sur la physique quantique? Elles m'ont convaincue. Ce qui tombe bien, car étant donné la compétence hors pair de Bitbol en physique, j'aurais éprouvé quelque embarras à m’y opposer en détail. La description du "vécu» de l'absolu? Il me faudrait, et ce serait trop long, dire à partir de quel point de vue je puis, sans arbitraire, opposer un vécu à un autre. Son interprétation de Kant? Mais Bitbol ne se prétend pas commentateur. Qu'importe donc si le portrait de Kant, non plus en vieux chinois mais en pragmatiste américain (voir plus bas), m'a quelque peu chiffonnée?

Bref, n'ayant trouvé de salut dans l'analyse d'un thème particulier, il ne me restait, hormis me taire, qu'à considérer la totalité. Avant qu'une franche hilarité ne gagne le lecteur à l'annonce d'un tel programme, sans doute faut-il spécifier, à ma décharge, que la philosophie de Bitbol se présente comme un enchaînement de raisons étroitement liées entre elles, et non comme une rapsodie de vues changeantes. Cette circonstance atténuante plaidée, je tacherai, tout d'abord, de mettre en lumière la structure du système, en faisant surgir les gestes les plus généraux, et les principes fondamentaux qu'ils induisent, pour exposer l'articulation entre les moments principaux de sa philosophie. Je le ferai en comparant son architectonique à celle de Meillassoux, afin de montrer la position de chaque joueur. Une fois cette vue en plongée effectuée, je m'intéresserai, par un brusque changement d'axe de la caméra, à un point de détail du livre de Bitbol, un minuscule grain de sable, mais susceptible, par la suite, de provoquer des objections en chaîne. Pour le dire d'un mot qu'éclaireront les analyses suivantes, l'essentiel de mon objection portera sur l'articulation entre philosophie réflexive, théorie de la connaissance et endo-ontologie. 


\section{L'architectonique des systèmes}

Les deux systèmes se laissent aborder à partir de trois mots que revendique Bitbol, et qui s'opposent à ceux qui qualifient la position de Meillassoux.

Transcendantal/ Réaliste. "Transcendantal» désigne l'enquête qui se dirige non vers les objets mais sur notre mode de connaissance des objets. Pour autant, Bitbol n'entend pas dévoiler des formes subjectives a priori et pérennes qui imposeraient de l'extérieur leurs normes à un matériau inchoatif, mais montrer comment tout objet connu est la cristallisation de l'interaction réciproque d'un pôle subjectif et d'un pôle objectif. Cette thèse épistémologique est établie à partir d'une analyse de la pratique scientifique, notamment de la physique quantique qui se laisse expliquer non comme connaissance d'un fait indépendant de nos modes d'accès, mais comme calcul visant à établir la probabilité de nos anticipations, sortes de paris qui, eux-mêmes, prennent sens au sein d'un complexe de techniques à notre disposition à un moment de l'évolution scientifique.

C'est donc par la notion d'action réciproque que Bitbol entend résoudre l'énigme de la connaissance: d'où procèdent les connexions que nous établissons entre les phénomènes, connexion comme la loi de la cause et de l'effet, ou connexion des différents moments du temps par la position de la permanence? Entre une réponse (globalement réaliste) qui affirmerait que c'est l'objet en soi qui informe (aux deux sens du terme) nos énoncés et une réponse (réputée idéaliste) qui attribuerait ces connexions aux compétences universelles d'un sujet connaissant, Bitbol montre comment ces "structures légales surgissent du jeu d'action et de réaction ${ }^{1}{ }^{»}$ entre une partie du monde et un sujet à la fois situé, immergé et agissant en son sein.

Ce geste de mise en relation réciproque s'oppose au geste de position d'un en soi par soustraction, qu'exemplifie le parcours de Meillassoux. En effet, une partie de sa démarche consiste, sur la base d'un objet admis comme connu (par exemple les énoncés "ancestraux» des sciences expérimentales), à en soustraire nos modes d'accès et ensuite à poser le " reste " ainsi obtenu comme "réel », soit ce qui, antérieur et postérieur à nous, en est indépendant, non lié, étymologiquement: ab/solu.

Ces deux gestes configurent le monde de chacun des protagonistes: un «grand dehors» ou monde sans nous pour le réaliste, des séquences de phénomènes, expressions de l'imbrication du sujet et de l'objet, pour le corrélationiste transcendantal.

Réflexif/Spéculatif. Réflexif traduit le mouvement de retour vers la source du connaître et s'oppose au mouvement d'aller vers un X, mouvement cette fois transitif, car se dirigeant vers une cible. Pour le dire d'un exemple: dans un acte de parole, le «je dis ", n'est pas ce dont on parle, la cible dont on dit 
quelque chose, mais ce à partir de quoi quelque chose peut être dit. Le réaliste effectue donc un mouvement de transitivité vers "ce dont on dit quelque chose », chose en soi qu'il prétend, au terme de son parcours, «intelliger». Tel est le sens ultime du terme "spéculatif ", qui désigne une philosophie en capacité de contempler, à partir du raisonnement, les propriétés de la chose. La philosophie réflexive, en revanche, suspend tout contenu X de l'objet (ce qu'on en dit) pour retourner à l'acte de le dire. L'opérateur qui permet ce retour vers la source est l'épochè husserlienne comme mise à distance d'un contenu et suspension de ses implications ontologiques.

Ce mouvement inverse commande la nature du principe ultime de chacune des philosophies: la nature en soi, chaotique et nécessairement contingente de Meillassoux, le «je maintenant et ici» de Bitbol. Parce que tout contenu énoncé suppose une énonciation proférée hic et nunc, le «je au présent de son énonciation" devient principe ultime que l'on ne peut nier sans le réintroduire sans cesse. Alors que la nécessité de la contingence de toute chose était le principe du réalisme spéculatif, cette fois, contingence et nécessité sont transférées au «je ", éprouvé dans le moment présent.

Phénoménologique/Scientifique. Pour Meillassoux, la science est habilitée à dire l'être. Mais que fait la philosophie si l'ontologie est assumée par la science? Meillassoux ne peut répondre ici qu'en concevant la philosophie elle-même comme "scientifique ", c'est-à-dire comme exercice rationnel qui délivre un savoir positif ( «la nécessité de la contingence») à propos de ce qui est indépendant de nous ( «le grand dehors»). Dans ce cadre, la science est absolutisée en un triple sens: d'une part, l'enquête philosophique part du contenu «littéral» des énoncés scientifiques; d'autre part, c'est une science, les mathématiques, qui permet de déterminer la structure de toute chose; enfin la philosophie délivre par voie rationnelle (et non sensible, religieuse, etc.) des contenus positifs sur les choses en soi. À l'opposé de ce geste d'absolutisation, Bitbol relativisera la connaissance scientifique. Relativisation signifie délimitation, et non, dénonciation. Il s'agit de situer la science en circonscrivant ses limites et, par là, sa sphère de compétence. Cette relativisation de la connaissance scientifique est rendue possible par l'usage de la méthode phénoménologique, qui intervient dans le système de Bitbol à deux niveaux: tout d'abord, l'épochè permet de développer la théorie de la connaissance, sur la base de l'analyse des pratiques scientifiques, en suspendant la prétention ontologique de leurs contenus objectifs. Mais la phénoménologie est aussi ce qui, une fois cette épistémologie établie, ouvre sur un autre domaine: l'endo-ontologie. Cette autre partie de la phénoménologie entend non pas connaître, en l'objectivant, l'être ou absolu, mais plutôt approcher son "vécu". Nous assistons donc à une fondation de la théorie de la connaissance par le haut et par le bas. Par le haut: l'Epochè, comme pratique réflexive, est le point de vue à partir duquel la connaissance scientifique est analysée; par le bas: l'enquête dévoile un champ d'investigation, celui du « je ici et maintenant », immergé dans une totalité dont il participe. 
La doctrine épistémologique de Bitbol, qui répond à la question «que fait la connaissance? " est ainsi bordée d'un côté par une philosophie réflexive et transcendantale, de l'autre par un type de parole (ou plutôt d'attitude), qui ne relève plus de l'acte de connaître mais dévoile un nouveau domaine: celui d'une ontologie d'immersion où il s'agit non pas de connaître, mais d'éprouver l'absolu.

Cette brève reconstruction de l'ossature des systèmes atteste, certes, de l'irréductibilité des gestes intellectuels effectués: relation réciproque / position par soustraction; mouvement réflexif de «retour sur» / transitif « en direction de "; absolutisation d'un X / relativisation des différentes composantes. Mais par delà ces différences qui rythment toute querelle corrélationisme / réalisme (et ses lignes de dégénérescence: relativisme / fondationalisme) se décèlent des points curieusement communs. Par exemple, dans l'opposition Transcendantal / Réalisme, les deux systèmes acceptent la possibilité que la nature soit chaos. Bitbol, en effet, considère cette thèse comme possible (mais non effective) puisque sa théorie de la connaissance repose sur l'affirmation que nos connexions sont présomptives. De même, l'opposition Réflexif / Transitif révèle que le principe ultime (la chose en soi ou le "je» de l'instant présent) partage une commune caractéristique: il est «nécessairement contingent ». Dans la troisième, ce qu'on dit de l'ontologie est dépendant d'une conception de la science. C'est évident chez Meillassoux, mais également tangible chez Bitbol: c'est parce que la connaissance scientifique peut, grâce à l'épochè, être limitée dans ses prétentions qu'elle ouvre sur le monde de l'endo-ontologie. En un mot: «Je dus donc limiter le savoir, pour faire place à l'éprouvé que prend en charge l'endo-ontologie.»

C'est sur ce problème de l'articulation, chez Bitbol, entre théorie de la connaissance scientifique et travail philosophique que je m'attarderai. Dans son architectonique, la conception de la connaissance occupe une place centrale, littéralement celle du milieu. Dès lors, si la théorie de la connaissance est problématique, tout le reste peut le devenir. Dit d'une analogie: qu'advient-il de l'édifice kantien si l'on prouve que la limitation du savoir effectuée dans la Critique de la raison pure est caduque?

\section{Un minuscule grain de sable}

C'est le rôle du philosophe, nous dit Bitbol, de suspendre son jugement sans s'appuyer sur tel contenu supposé définitif d'une science particulière. L'épochè requiert neutralité et table rase, mots utilisés dans son livre, qui retrouve la radicalité de Husserl: pas de présupposés. Et assurément, Bitbol y parvient magnifiquement en analysant les théories physiques. Néanmoins, il reste un contenu scientifique qui, jamais frontalement interrogé, traverse le livre. S'y trouve, en effet, nombre de passages que je qualifierai, au plus court, de darwiniens, comme: «Prise au sens de la révolution copernicienne, la connaissance humaine n'est en somme rien de plus qu'une étape avancée, parce qu'universalisée et formalisée, de la poussée adaptative initiée par 
l'évolution des espèces » (69). De même, reprenant à son compte une partie du QBism, il considère l'ordre apparent que nous conférons aux phénomènes comme "un système [...] de paris, grâce auxquels les agents macroscopiques organisent par avance les conditions dynamiques de leur survie au cœur d'un environnement qui pourrait être chaotique» (241). Ces paris sont, non pas des possibles imaginairement construits par une raison autonome et hors sol, mais des anticipations "vitales». La mobilisation de la théorie énactive de la relation sujet-objet permet d'affirmer que le sujet connaissant est un organisme qui tire des "saillances signifiantes» de son environnement disponible "afin de s'orienter et de survivre en utilisant ses moyens sensori-moteurs limités» (297). Bref, le souci de la science d'établir des connexions n'est autre qu'un "besoin vital de légaliser ce qui arrive» (192).

Que le lecteur se rassure, je n'entends pas contester la thèse de l'évolution mais simplement noter que: 1) eu égard à la méthode promue par Bitbol, il semble que cette thèse (qui dépend d'une science constituée) devrait non pas être présupposée, mais subir le même traitement que les théories physiques: suspension de leurs implications ontologiques, réduction de leur savoir à la pratique de paris organisateurs à partir d'un fond possiblement chaotique. Mais plus encore: 2) quand bien même on accepterait cette thèse, en quoi est-elle susceptible d'expliquer l'homme et plus spécifiquement son acte de connaître? L'homme, produit de l'évolution adaptative, agit en vue de sa survie. Sans doute, mais et alors? La chauve-souris et les virus aussi. Dès lors, on ne voit guère ce que cette référence à l'évolution explique en matière d'épistémologie, sauf à adhérer à une version darwinisée du mythe d'Épiméthée: l'homme, créature pitoyablement finie et oubliée des dieux, n'aurait trouvé d'autres moyens d'assurer son maintien qu'en développant cette habileté saugrenue, la connaissance.

Or cette référence à l'évolution a des implications en chaîne sur l'ensemble du système. Partant d'une analyse de la physique, généralisant les résultats acquis à la connaissance scientifique puis à la connaissance théorique tout court, Bitbol finit par réduire l'acte de connaître en général à un calcul visant à évaluer le succès de la variété des pratiques que nous pouvons réaliser dans certains contextes limités; pratiques qui elles-mêmes sont initiées - cause efficiente - et vectorisées - cause finale - par un besoin vital. La finalité de toute connaissance théorique devient l'utilité, en son sens le plus trivial, pragmatique et biologique.

La relecture pragmatique de Kant entreprise dans le chapitre V va dans ce sens. Cependant, au fil du livre, l'usage du terme «pragmatique» se révèle équivoque, car il fait se chevaucher deux significations pour le moins dissemblables. La première acception renvoie à l'usage du terme "pratique » par les post et néo-kantiens (Fichte, Lask) et réfère à une praxis purement intellectuelle, où l'acte est entendu comme accomplissement (prasso: accomplir; aller jusqu'au bout en passant à travers). La seconde réfère à une certaine tradition pragmatique américaine et thématise l'action matérielle 
(pragma: action, affaire; pragmaticus, relatif aux affaires), tradition pour laquelle l'homme est un organisme dont toutes les actions (manger, aimer, connaître) ont pour fin la lutte efficace pour la survie et l'intérêt personnel.

Fichte, sur lequel Bitbol s'appuie à de multiples reprises, illustre la première manière de comprendre la proposition: la pensée est acte. Penser, c'est accomplir un certain nombre de gestes cognitifs (par exemple poser A, lui opposer B, les mettre en relation) mais ces actes n'ont pas pour finalité une quelconque utilité, fût-ce celle, vide à force d'être vaste, de survivre. Si le savoir est une praxis, c'est parce qu'il est une concaténation d'actes intellectuels et non d'actions matérielles visant la maitrise, excroissance de la prédation. Certes, chez Fichte, cette série d'actes intellectuels vise un accomplissement: la réalisation de l'universel, lequel est une idéalité, qu'on ne trouvera nulle part dans la nature (qu'elle soit comprise comme monde extérieur ou comme complexion biologique). Cette réalisation de l'universel est expression de la liberté (d'où le terme: pratique). De cette finalité librement posée et non causalement suscitée par notre condition biologique, découle, en retour, un certain nombre de normes idéales à performer, telles: ne pas annuler le contenu de ce que l'on dit par l'acte de le dire, ne pas rester au niveau de la position d'un $\mathrm{X}$ dans son opposition à -X, sans chercher, dans une entente supérieure, à les mettre en relation, etc. Ces normes qui régissent la praxis théorique sont celles de la vérité épistémique, laquelle se définit comme réalisation de l'universalité. Dans ce cadre, la vérité, pour ne pas se définir comme duplicata d'un être extérieur (refus du réalisme comme chez Bitbol) ou comme simple cohérence formelle, ne peut, pour autant, se rabattre sur la simple utilité. Ne serait-ce que parce que la conception d'une vérité comme utilité ne résout en rien le problème de la connaissance. Car enfin, qu'est-ce que l'utilité ? L'utilité pour moi, ma tribu, ou encore la terre? Mon intérêt (ou le nôtre, à supposer qu'il y ait compatibilité) sera-t-il défini à court ou à long terme? Qui décidera de ce qui est utile? Le problème classique de «l'autorité épistémique» demeure entier.

Par le chevauchement problématique des termes "pratique» (actes intellectuels) et «pragmatique» (actions sur X, matériellement ou corporellement efficaces), l'épistémologie de Bitbol, et la conception de la vérité qui la soutient, majore l'utilité et occulte, sans justification ni discussion frontale, l'universalité.

Différents autres indices en témoignent. Ainsi, dans la proposition déjà citée, page 69, le processus d'universalisation apparaît comme un instrument au service de l'utilité. Il prend en outre la forme d'un pari inductif, dont la probabilité de succès est encadrée par le plus et le moins. C'est cette probabilité qui permet d'attribuer à une théorie sa plus ou moins grande vraisemblance: la probabilité que tel virus calamiteux disparaisse par intervention divine est, tout bien calculé, moins grande que par vaccin. Mais ce simple exemple indique que ce sont nos croyances du moment, soit nos habitudes acquises (nous sommes plus scientifiques aujourd'hui que reli- 
gieux), qui sont affectées d'un coefficient de probabilité. En quoi a-t-on dépassé le problème de Hume ou conjuré le risque du relativisme?

L'indique pareillement sa conception des mathématiques, lesquelles paraissent un outil au service de la physique. Or, quand bien même on accepterait les analyses hautement instructives de Bitbol sur la physique quantique (ce que je fais), comment ne pas penser que les mathématiques sont une discipline à part entière qui a des procédures de vérité et d'universalisation qui semblent peu réductibles à la théorie des paris? Une prise en considération des procédures de vérité propres aux mathématiques ne seraitelle pas susceptible de fragiliser cette théorie de la connaissance qui paraît, in fine, substituer la vraisemblance ponctuellement efficace à la vérité? Bref, Bitbol paraît, au fil de ses analyses, interpréter toute connaissance théorique comme simple croyance affectée d'un plus ou moins grand degré de probabilité et rabattre toute idéalité (fût-elle affaiblie comme chez Kant en idéal régulateur) sur une sorte de compétence naturelle: "nos anticipations sont un besoin vital»(255), nous dit-il, pour résoudre l'énigme du vleu de Goodman, mais ne sont que des croyances vraisemblables.

Certes, on peut comprendre les écueils que Bitbol a voulu éviter en réduisant ainsi la connaissance théorique: celui du réalisme (cible du livre) mais aussi l'aspect rigidement fixiste du kantisme (structures pérennes d'une subjectivité universelle) ou encore l'épineux problème, chez Husserl, du statut des idéalités universelles (réalisme des essences?). Mais ces difficultés justifient-elles de rabattre toute idéalité sur un calcul des coûts (ou des coups pour la théorie des jeux)?

Bitbol paraît donc procéder à une limitation excessive de la connaissance théorique, dont les accents font parfois songer à la critique heideggérienne de «l'arraisonnement théorique». N'y avait-il pas lieu d'envisager d'autres possibilités: celle par exemple de la tradition rationaliste qui, avec la notion d'universalité, démontre que la discussion sur la définition de la vérité ne se limite pas à trois possibilités: réalité / cohérence / utilité ? La connaissance rationnelle se résume-t-elle à un travail d'entendement ou n'y a-t-il pas, au-delà de l'Erkenntnis kantienne, une place pour un savoir (Wissen), qui ne serait pas retombée dans le réalisme? Pour l'exprimer à partir de traditions différentes: une distinction ne doit-elle pas être faite entre un savoir "théorique» visant un gain autre que lui, et l'activité théorétique (le savoir pour le savoir) qui, chez Aristote, débouchait également sur la thématisation d'un acte purement intellectuel? Dans un tout autre ordre d'idée, on a pu développer ces derniers temps (Engel, Pouivé) une réflexion sur les vertus épistémiques, sans pour autant affirmer que ces vertus sont les mêmes dans n'importe quel champ d'action (ordinaire, technique, économique). La sphère épistémique peut être une sphère autonome, et les actes qui la composent ne pas être de simples exemplifications de l'action concrète et de la décision efficace. 
Ce déluge de références à des traditions multiples n'a pas pour but de les opposer de manière externe à Bitbol en déclarant arbitrairement qu'une telle a ma préférence, mais bien de montrer que l'équation: connaissance en physique = objectivation utile = connaissance théorique en général, ferme, sans s'en expliquer, d'autres voies pour penser la nature du savoir théorique et sa notion cardinale: la vérité.

Or c'est cette limitation problématique de la connaissance qui détermine l'ouverture à l'endo-ontologie et permet de définir, par négation, le type de parole qui y sera adéquat: parole non de connaissance théorique, mais d'attestation, non de raison, mais de vécu éprouvé ici et maintenant. J'envisagerai tout d'abord la transition problématique entre connaissance théorique et type de discours propre à l'ontologie, pour ensuite me tourner vers le contenu de cette endo-ontologie.

\section{De la rationalité à l'éprouver, la conséquence est-elle bonne?}

Parce que cette ontologie d'immersion ne relève plus de l'activité théorique et rationnelle, mais de "l'éprouver ", le type de parole qui l'appréhende sera comparé au mystique du Tractatus. Nous passons du théorique au vécu, du rationnel au "saisissement actuel de l'être là ». Ce passage était-il le seul possible? Disons-le d'une analogie: on se souvient du fameux portique des Méditations de Husserl, que Descartes ne franchit pas car il objective l'ego. Ce qu'on ne saurait reprocher à Bitbol, qui s'en tient au seul "je» existentiel, pur éprouver dans l'instant, qui n'a ni passé (l'identité numérique est renvoyée au sujet empirique) ni futur. Cependant, le portique de Husserl n'ouvrait pas sur le seul «je» proféré et vécu, lequel stricto sensu était le portique même: porte d'entrée et non point d'arrivée. Il dévoilait un nouveau monde, celui des idéalités, structures universelles de l'apparaître justiciables d'un traitement rationnel. Les appréhender ne nécessitait nullement de proclamer la faillite de tout discours théorique au profit d'un indicible, même "se montrant». Entendons-nous bien, il ne s'agit pas de reprocher à Bitbol de ne pas être totalement husserlien (c'est son droit) ni moins encore de contester ses magnifiques descriptions de tel vécu particulier, mais de suggérer que cette endo-ontologie, parce qu'elle repose, au départ, sur une limitation excessive de la connaissance théorique, s'apparente parfois au salto mortale de Jacobi. Soudainement, foin de survie et de calcul de coûts, propres à l'activité théorique, l'homme se fait éprouvant et contemplatif, au sens des spiritualités religieuses. D'autres possibilités étaient peut-être envisageables, d'autant que pareille promotion d'un saisissement «muet » posera inévitablement la question du passage du « je » au nous (l'universel, encore!).

Outre cette transition, moins nécessaire qu'elle n'y paraît, à l'endoontologie, son contenu propre fait problème. Mais comment, se demanderat-on, parler d'un contenu de l'onto/logie, si le type de parole qui y est pertinent ne relève plus du logos? Plus préoccupant encore, non seulement l'ontologie a un contenu, mais ce dernier deviendra par un mouvement en 
retour, le sol originaire de tout l'édifice (du savoir théorique comme des énoncés «suspensifs» initiés par l'épochè). En fait, le schéma suivi par Bitbol, en apparence circulaire, ne l'est pas. Le type de parole propre au champ de l'endo-ontologie n'est découvrable qu'à partir de la relativisation de la connaissance, mais en retour les contenus de l'endo-ontologie se révèleront le socle, la base, le sol de toute certitude. Toute action, réflexion, pensée, connaissance, deviendra relative au principe suprême, le "je vécu en son présent vivant". Il n'y a pas cercle vicieux, car l'ordre du dévoilement du principe est rarement l'ordre de la fondation. Dit d'un exemple: Descartes, à la différence de Spinoza, ne pose pas en premier le fondement de son système (Dieu en l'occurrence), mais le dévoile au terme de la mise en œuvre d'une certaine méthode. Dieu est néanmoins premier, et on montrera ensuite que tout ce qui précédait lui était relatif. Je pourrais donc, si je me faisais l'opponens de Descartes, soit contester que la méthode utilisée conduise nécessairement à Dieu et non à autre chose (question sur la légitimité du passage) soit révoquer le contenu même du fondement: Dieu n'est pas ce qu'il en dit. De même, ayant critiqué, chez Bitbol, la légitimité de la transition (pourquoi passer à une parole non plus rationnelle, mais existentielle?), je dois maintenant considérer le contenu de cette parole, que j'aborderais à partir des trois notions, qui, à mon sens, en sont le soubassement, à savoir : Être, Totalité, Présent.

Être. La plénitude d'être est clairement définie comme le lieu en lequel et au-delà duquel aucune négation n'est possible. Toute détermination étant négation, déterminer serait transformer la pure quoddité («il y a ») en aliquid, c'est-à-dire en "quelque chose ", dont la définition implique la négation de ce qu'il n'est pas. Mais si l'être est le néant de toute détermination, pourquoi est-il appelé «être» plutôt que "vide» ? Pourquoi choisir la solution "Heidegger» (Être / ontologie) plutôt que le vide de certains bouddhistes, qui implique un abandon de toute ontologie, fût-elle d'immersion? Et comment pouvons-nous discuter de ces deux possibilités égales (être ou vide? reconduction ou refus de l'être?) si nous ne nous appuyons que sur notre propre "vécu », cet espace où le discours n'est plus de mise?

Nous pourrions dire la même chose à propos de la "stupéfaction d'être ", le saisissement du "c'est ainsi ", stupéfaction qui est posée comme première et originaire, car elle ne s'autorise d'aucune pensée antécédente: "Cela pourrait être autrement, et c'est ainsi; mais la pensée que cela pourrait être autrement n'est que l'écho affaibli et abstrait de la feérie de son: "c'est ainsi" "(451). Pourquoi ne pas dire l'inverse: la stupéfaction du "c'est ainsi» n'est possible que par considération de l'idée (quel autre mot moins désuet choisir ? possibilité conceptuelle?) que cela aurait pu être autre? La stupéfaction "que cela soit " semble ne pouvoir être caractérisable comme surprise qu'à condition de posséder la notion de "aurait pu être autrement». Dès lors pourquoi, là encore, maintenir le privilège de l'ontologie au détriment d'une philosophie qui secondariserait l'être et investirait 
la notion de «possible» que dessine la notion de "pouvoir être autrement »? Stricto sensu: pourquoi une onto/logie (discours sur ou de l'être) et non une méta/physique (au-delà de ce qui «est ainsi»), qui ne serait plus amarrée à l'être?

Totalité. La plénitude est définie, avec Merleau-Ponty, comme immersion dans la totalité. Par manque de place, je ne m'attarderai pas à la question "d'où sort-on cette catégorie de la totalité?", ce que je pourrais néanmoins faire, non pour accuser à tort Bitbol de reprendre des catégories de la métaphysique classique, car chez lui elles ne renvoient pas à un être transcendant, mais simplement parce qu'une enquête réflexive peut se demander par quels processus un sujet irrémédiablement situé et fini en arrive à former des notions comme totalisation (ou universalisation, infinitisation, etc.). La question que je poserai est plus bête: "Pourquoi vouloir l'immersion? » Puisqu'on est au niveau du vécu, pourquoi n'opposerais-je pas un autre vécu (le mien ou celui puisé dans mille milliards de poèmes) en affirmant tout de go que, loin d'être candidate à la noyade dans le grand tout de l'être (la fusion, l'immersion), je considère cet état comme le fond de l'horreur et non de la plénitude? Car enfin, s'il s'agit du vécu du «je ici et maintenant », comment parviendrons-nous à en débattre? À cette question, trois réponses semblent possibles:

1) ou bien nous reconnaissons la singularité de l'expérience et admettons que nous ne pouvons en discourir. Ce n'est pas une onto-logie que nous proposerions, mais l'attestation d'un vécu singulier.

2) ou bien l'un d'entre nous, du fait de sa position dominante, qu'il s'agisse d'une autorité sans justification (l'autorité épistémique supposant l'argumentation) ou de la référence à une majorité ou alors à un Plain man (Austin), érigera son expérience en paradigme, qui deviendrait alors la norme sur le mode: vous n'avez pas cette expérience mais vous devriez; vous vous révélez donc, par votre objection, handicapé de la fusion. Mais au nom de quoi une expérience peut-elle être dite paradigmatique de toute autre et se transformer en norme, comme on le voit exemplairement dans les philosophies de l'ordinaire, où telle expérience X (par exemple la perception) est posée en paradigme de toute expérience sensorielle et érigée ainsi en norme, par rapport à laquelle l'hallucination sera considérée comme déviance (inusuelle, anormale, nous dit Austin) ? Mais, en aucun cas, Bitbol, si attentif aux expériences limites, qu'il valorise dans tous ses livres, ne peut adhérer à pareille réponse. Dès lors, si ni 1 ni 2 alors:

3) nous pouvons en discuter à partir de la considération de la divergence de nos vécus. Mais, ce faisant, nous dessinerions un tout autre schéma, où nous dirions: nous ne pouvons rétrocéder à un éprouvé universel (la plénitude d'être). Tout logos surgit non d'une expérience identique, mais de la différence. C'est à partir de cette différence que nous pouvons commencer à discuter pour nous élever à un commun, qui, ni base ni sol, est un idéal. 
Où nous retombons sur l'universel, qui ne sera jamais trouvé ni dans la nature ni dans notre vécu, car il n'est pas «donné», mais à réaliser.

Le présent, la présence. Le «je» en son présent vivant est l'ultime principe sur lequel tout repose. Tout lui est relatif, nous dit Bitbol. "Sanctuaire de la certitude", il est cet instant bordé de deux néants, le passé qui n'est plus et le futur qui n'est pas encore. Bref, ce principe, "éprouvé ", socle de l'endo-ontologie, est également le seul fondement des connaissances théoriques, qui ne connaîtraient pas de certitude d'un autre type, car, pour ce qui est de leur contenu, elles ne sont que présomptions. Toute certitude est-elle relative au présent?

Assurément, la certitude du «je au présent vivant» peut valoir pour le Cogito cartésien, qui relève de la non-contradiction pragmatique (je ne puis dire l'inverse sans le réintroduire), mais aussi et fortement de la dimension du maintenant («à chaque fois que je le prononce»). Mais ce présent brut vaut-il pour toute contradiction pragmatique? Soit une proposition classique à partir de laquelle s'exemplifie également la contradiction: «la vérité n'existe pas». C'est une contradiction, car l'énonciation (le faire) annule le contenu de l'énoncé (le dire). Or, pour établir la contradiction, il faut admettre un léger différé entre mon dire "la vérité n'existe pas" et la conscience de mon faire qui invalide son contenu: "Je réalise que, disant cela, je présuppose qu'il y a au moins une vérité. » Je dois donc me souvenir de mon dire qui est prononcé avant que je ne puisse le révéler comme contradictoire, et donc incertain. La première proposition appartient à un presque présent mais néanmoins passé. La contradiction entre le dire et le faire se conçoit dans la succession (je dis X et m'aperçois que Z). Qu'en conclure? Que ce type de contradiction pragmatique n'a aucune certitude ou que le temps de la certitude n'est pas seulement pur présent bordé de néant? Dans notre exemple, la certitude repose sur un choc entre "passé immédiat» et «présent ». Il conviendrait donc de s'interroger sur la notion de succession. Doit-elle s'interpréter en termes de passé / présent / futur ou en termes d'enchaînements discursifs? Et si oui, de quoi relèvent ces enchaînements? Du temps (la notion de succession, de manière obvie, renvoie à l'avant et à l'après) ou de l'espace ( «être le successeur de» peut se concevoir sur une ligne tracée à partir d'un point zéro), ou encore d'un enchaînement hors considération de temps et d'espace? Auquel cas, la certitude naîtrait d'un enchaînement seulement rationnel. "Essayez de sortir du maintenant", ce défi se relève peut-être par l'acte de penser X et de savoir qu'on le pense.

On objectera que la certitude qui naît de la contradiction se fera au présent. Certes, mais la condition de son advenue est que j'ai le souvenir d'un passé immédiat. On nous rétorquera encore que tout cela mobilise quand même un «je». Assurément, mais de quel «je» s'agit-il ? Du «je» plein de la présence éprouvée? Il paraît plutôt relever de l’Ichspaltung de Husserl, ce «je » qui ne surgit que par et dans la scission qu'il opère entre un vécu et son examen, un énoncé "dit» et son acte d'énonciation. Le "sum» 
ici ne se définit pas par l'être, sa participation à la totalité et sa présence dans l'instant, mais par sa division, condition de l'épopée réflexive.

Or, comme pour l'usage de "pragmatique, le «je» chez Bitbol fait se chevaucher deux significations: la première renvoie uniquement à l'acte d'énonciation: tout énoncé suppose un "je dis que ", que l'on ne saurait nier sans le présupposer à nouveau. Mais à en rester à ce niveau il n'y a aucune raison de lester ce «je»d'un quelconque contenu. En effet, je ne puis faire retour sur lui et le prendre comme objet dont je pourrais énoncer les caractéristiques puisque le «je » est la condition toujours impliquée. Si tout lui est relatif, c'est au sens où on ne peut pas ne pas le présupposer (argument de type transcendantal).

Mais Bitbol ne se contente pas de ce niveau que je qualifierai de logique au sens large de: ce qui s'établit par un argument relevant du logos et qui se laisse, dès lors, caractériser comme acte discursif, soit, ici, comme savoir d'impossibilité: je prends acte qu'il est impossible de nier X. Ce premier «je» principiel devient, chez Bitbol, un être vécu dans le milieu de présent vivant, participant à la totalité (chair ou être) et «sanctuaire de certitude». Mais si on accepte nos analyses précédentes, elles semblent attester qu'il existe des types de certitudes à même le discours rationnel lui-même sans qu'il ne soit besoin de mobiliser un «je éprouvé». Ce dernier est certes possible, et il ne s'agit nullement, pour moi, d'en refuser arbitrairement les approches. La philosophie doit bien sûr accepter les expériences de tout ordre, qu'il s'agisse des méditations sotériologiques des spiritualités bouddhistes ou des pratiques artistiques. Il s'agissait juste de montrer que la réduction de toute certitude au présent vivant n'est pas justifiée. On saute trop vite du rationnel au mystique (par limitation excessive du premier), et le type de certitude que dévoile l'endo-ontologie ferme, sans la discuter, la possibilité qu'il existe d'autres certitudes qui n'appellent pas une sortie de la discursivité rationnelle.

Pour achever trop rapidement cette réflexion sur le "présent vivant ", posons une dernière question, d'apparence intempestive: les trois temps invoqués par Bitbol (passé, présent, futur) sont-ils les seuls à notre disposition? Loin de moi l'idée de me mêler des querelles des linguistes, qui aboutissent aujourd'hui à classer majoritairement le conditionnel non plus parmi les modes mais parmi les temps. Temps étrange qui semble mobiliser futur, présent et passé. Quoi qu'il en soit, philosophiquement considéré, ce temps opère une sortie hors du fait objectivé (qui appartient, pour Bitbol à la connaissance théorique), mais tout autant hors de l'être, du vécu, de la présence (qui relève de l'endo-ontologie). Serait-ce le temps de la pensée théorétique, celle des possibles où la question n'est plus ce qui est, ni comment je le vis? Ces possibles ne sont-ils pas susceptibles de vérité, à condition qu'on cesse de la définir à partir d'une "réalité originaire» de l'être ou du vécu? Nous revenons à une objection antérieure: certes, je ne puis expérimenter le «pouvoir ne pas être» que parce que je suis maintenant. Mais ce je suis est-il 
expérimentable autrement qu'à partir de l'idée que je pourrais ne pas être? Loin d'être un écho affaibli et abstrait du "c'est ainsi ", le conditionnel du "pouvoir ne pas être» est peut-être le temps de la raison, par laquelle nous sortons à la fois du présent et de l'être, pour aller au-delà, vers des mondes possibles, dont la vérité est susceptible d'être établie par le biais d'une réflexion sur ce qui, in fine, me semble le grand absent de chaque niveau du système de Bitbol: l'universel.

La disputatio imposait d'objecter, je me suis pliée à la règle du jeu. J'ai décomposé les raisons pour lesquelles le refrain d'une chansonnette n'a cessé d'accompagner ma réflexion sur ce très grand livre: "Y a quelque chose qui cloche là dedans. » À l'instar du tonton loufoque de Boris Vian, je ne puis que conseiller au lecteur et à ses neveux de conclure d'eux-mêmes, en chantant: «J’y retourne immédiatement!» 